\title{
Detection of [O I] $\lambda 6300$ and Other Diagnostic Emission Lines in the Diffuse Ionized Gas of M33 with Gemini-North
}

\author{
E. S. Voges and R. A. M. Walterbos \\ Astronomy Department, New Mexico State University, Las Cruces, NM 88003; \\ esgerken@nmsu.edu, rwalterb@nmsu.edu
}

\begin{abstract}
We present spectroscopic observations of diffuse ionized gas (DIG) in M33 near the H II region NGC 604. We present the first detection of [O I] $\lambda 6300$ in the DIG of M33, one of the critical lines for distinguishing photo- from shock ionization models. We measure $[\mathrm{O} \mathrm{I}] / \mathrm{H} \alpha$ in the range of 0.04 to 0.10 and an increase in this ratio with decreasing emission measure. Our measurements of $\left[\begin{array}{ll}\mathrm{S} & \mathrm{II}\end{array}\right] / \mathrm{H} \alpha$ and $[\mathrm{N} \quad \mathrm{II}] / \mathrm{H} \alpha$ also rise with decreasing emission measure, while our $[\mathrm{O} \mathrm{III}] / \mathrm{H} \beta$ measurements remain fairly constant. We have one tentative detection of He I in the region of brightest emission measure, with a ratio of $\mathrm{He} \mathrm{I} / \mathrm{H} \alpha=0.033 \pm 0.019$, indicating that the helium is at least partially ionized. We compare our observed emission line ratios to photoionization models and find that field star ionization models do not fit our data well. Leaky H II region models are consistent with our data, without the need to invoke additional ionization mechanisms to fit our [O I] or [O III] measurements. The closest large H II region is NGC 604 and is therefore a likely candidate for the source of the ionizing photons for the gas in this region.
\end{abstract}

Subject headings: galaxies: individual (M33) — galaxies: ISM

\section{Introduction}

The influence of massive stars and their deposition of energy into the interstellar medium (ISM) can be seen through their creation of H II regions, superbubbles, filaments, and chimneys. The full effect of massive stars' presence in galaxies is still unknown, but they are thought to also be responsible for the diffuse ionized gas (DIG, or warm ionized medium, WIM) that has been observed in both the Milky Way and external galaxies (e.g. Reynolds 1991; Rand et al. 1992; Greenawalt et al. 1998; Hoopes \& Walterbos 2003). In the Milky 
Way, the diffuse ionized medium is known as the Reynolds layer. This gas is warm $(8,000-$ $10,000 \mathrm{~K})$, has an electron density of $0.1-0.2 \mathrm{~cm}^{-3}$, and is the most massive component of the ionized Galactic ISM. Studies of external galaxies have shown the DIG in other galaxies to be similar in spatial extent to that of the Reynolds layer.

The Wisconsin H $\alpha$ Mapper (WHAM) Northern Sky Survey has revealed that the ionized gas in the Milky Way is detectable in almost every direction in the northern sky (Haffner et al. 2003). The total power required to ionize the local DIG in the Galaxy is about $4.0 \times 10^{-5}$ ergs s${ }^{-1} \mathrm{~cm}^{-2}$, if all of the gas is assumed to be at a temperature of $10^{4} \mathrm{~K}$ (Reynolds 1990). Only OB stars can meet or exceed this ionizing power requirement. Studies of external galaxies have suggested the power requirement from a galaxy's OB stars is around $40 \%$ to ionize the DIG (Hoopes et al. 1996; Ferguson et al. 1996; Greenawalt 1998), based on the observed DIG's contribution to the galaxy's $\mathrm{H} \alpha$ luminosity.

One way to test whether the power source of the DIG is photoionization by massive stars is to observe its optical spectrum. Numerous spectroscopic studies of the DIG in galaxies have revealed that DIG has enhanced [S II] $\lambda 6716 / \mathrm{H} \alpha$ and [N II] $\lambda 6584 / \mathrm{H} \alpha$ compared to H II regions (e.g. Ferguson et al. 1996; Hoopes \& Walterbos 2003). These emission line ratios are well replicated by pure photoionization models. However, there are other emission line ratios that are difficult to replicate and challenge pure photoionization models. Observations of edge-on galaxies have found that the $[\mathrm{O}$ III $] / \mathrm{H} \beta$ ratio may rise with distance from the plane (Rand 1998) at a level that photoionization models may not be able to reproduce. Other mechanisms suggested to create this rising $[\mathrm{O} \mathrm{III}] / \mathrm{H} \beta$ ratio include shock heating (Shull \& McKee 1979) and turbulent mixing layers (Slavin et al. 1993). These models have some support from observations that the [O III] spectral line can be broader than other spectral lines (Wang et al. 1997). The [O I] $\lambda 6300 / 6363$ doublet is another indicator of the relative contribution of shocks to the ionization of the DIG (e.g. Dopita 1976). These lines are difficult to observe in nearby galaxies due to the bright [O I] airglow line. Little information has yet been gleaned from this important emission line in face-on galaxies. Finally, little is known about the ionization fraction of helium versus hydrogen, which can be probed by the ratio of He I $\lambda 5876$ to $\mathrm{H} \alpha$. Due to many bright skylines near He I, there is insufficient reliable data available on He I emission in face-on galaxies. Only O7 stars or earlier produce the high-energy photons required to ionize helium, so the presence of He I emission can give also clues as to the type of stars responsible for the ionization of the DIG.

M33 is an ideal subject for spectroscopy of DIG because it is nearby (0.84 Mpc away) and is suitably inclined. M33 is blueshifted by $-180 \mathrm{~km} \mathrm{~s}^{-1}$ with respect to heliocentric velocities, making the observation of [O I] possible. To take full advantage of this small blueshift, we used Gemini's nod-and-shuffle capabilities to get the best sky subtraction possible. 


\section{Observations and Data Reduction}

The data were taken using the Gemini Multi-Object Spectrograph (GMOS) on the Gemini-North telescope by Gemini staff in the queue mode during 2005 January and February (as part of Program GN-2004B-Q-18). We obtained long-slit spectra with a slit 108" long and 1" wide of one position in M33 (see Figure 1). The target area is a region with an $\mathrm{H} \alpha$ emission measure of about $25 \mathrm{pc} \mathrm{cm}^{-6}$ near NGC 604 . We were concerned with obtaining accurate sky line subtraction, so we traded on-target time for frequent nodding off-target to get sky exposures. Three observing blocks of 3600 seconds open-shutter time were obtained. Each exposure had 15 nod-and-shuffle cycles (a complete cycle being one exposure of the science target followed by one exposure of the sky), with each cycle being 240 seconds long. This came to 1800 seconds on-target for each observing block. Our nods were 9.2 ' long, taken in the direction parallel to the slit.

The R831 grating was used with the three mosaiced EEV $2048 \times 4068$ CCDs, and the data were binned on chip by 2 in the wavelength dimension and by 4 in the spatial dimension.

The resulting dispersion scale was $0.68 \AA$ per pixel and the resulting spatial pixel scale was 1.19 parsecs per pixel. The spectral resolution measured on the arc lamp lines was $3.0 \AA$. This spectral resolution was lower than anticipated due to a focus problem. The grating was tilted to a central wavelength of $5858 \AA$, with coverage from about 4850 to $6860 \AA$.

The data reduction was carried out with the Gemini IRAF package. After bias subtraction and multiplication by the gain for both the science and sky spectra, the sky spectra were subtracted from their corresponding science spectra. The sky-subtracted spectra were then combined, mosaiced, and trimmed to include only the middle third of the CCD (i.e. where the science exposure was located). The combined spectrum was then flat-fielded, wavelength and flux calibrated, and corrected for atmospheric extinction. Standard star observations were included in our program, however, they were not observed in conjunction with our science observations. As a result, we do not have a good absolute flux calibration. However, we do have a good relative flux calibration, which is sufficient to compare emission line strengths.

Charge-traps in the GMOS CCDs were evident in our spectra. These charge-traps are visible as streaks in the horizontal, or wavelength dispersion direction. Abraham et al. (2004) suspect that these traps are due to small detector defects that are repeatedly "pumped" by the shuffle-and-pause action associated with nod-and-shuffle observations. Although the presence of these streaks is visually unappealing, we did extensive noise analysis of the CCDs both near to and far from these streaks, and the noise characteristics seemed unaffected by the charge-traps. We did, however, find that the overall rms noise for the middle of the three CCDs was about 1.6 times higher than the other two. This was unfortunate for us as the 
faintest of our desired emission lines (He I $\lambda 5876$ and [N II] $\lambda 5755$ ) fell on this CCD. The combination of high overheads (and thus reduced observation times), the intrinsic faintness of the lines, and a noisy CCD made it impossible for us to detect [N II] $\lambda 5755$, and we detected He I in only one aperture.

\section{Emission Line Ratios}

We present results on our detection of $\mathrm{H} \alpha,[\mathrm{S}$ II] $\lambda 6716,[\mathrm{~N}$ II] $\lambda 6583$, [O I] $\lambda 6300$, [O III] $\lambda 5007$, and $\mathrm{H} \beta$, as well as a tentative detection of He I $\lambda 5876$. We were unable to $\operatorname{detect}[\mathrm{N} \mathrm{II}] \lambda 5755$ or $[\mathrm{O} \mathrm{I}] \lambda 6363$. We detected $[\mathrm{S}$ II] $\lambda 6731$, but the ratio between the two [S II] lines is constant in low density gas, so we do not present any results for [S II] $\lambda 6731$. The emission line ratios presented here were determined by averaging the spectrum along the spatial direction over 42 pixels. At an assumed distance of $0.84 \mathrm{Mpc}$ to M33, this corresponds to apertures approximately $50 \mathrm{pc}$ in length. The $\mathrm{H} \alpha$ emission measure profile of the gas along the slit is fairly smooth, so we chose equal sized apertures that increased our signal to noise while preserving some spatial information along the slit. To find the uncertainties in the measurements, we multiplied the average rms noise on either side of each emission line by the square root of the number of pixels contained in the line. The individual uncertainties were added in quadrature for the uncertainty in the ratios.

\subsection{The $[\mathrm{S} \mathrm{II}],[\mathrm{N}$ II], [O III], and He I Emission Lines}

Figure 2 shows the ratios of $[\mathrm{S}$ II $] \lambda 6716 / \mathrm{H} \alpha,[\mathrm{N}$ II] $\lambda 6583 / \mathrm{H} \alpha,[\mathrm{S} \mathrm{II}] /[\mathrm{N}$ II], [O I] $\lambda 6300 / \mathrm{H} \alpha$, [O III $] \lambda 5007 / \mathrm{H} \alpha$, and [O III $] / \mathrm{H} \beta$, along with the underlying $\mathrm{H} \alpha$ emission measure profile. Given the location of the slit, the x-axes on these plots can be taken not only as distance along the slit, but also as increasing distance away from NGC 604. In general, the H $\alpha$ emission measure is seen to decrease with distance along the slit. If we are to assume that the density of the gas is about constant along the slit and that NGC 604 is a contributing source of ionizing photons in the region, then for a sufficiently thick disk of gas we might expect a roughly spherical region of gas centered on NGC 604 to be influenced by its ionizing photons. If that is the case, then a decrease in the $\mathrm{H} \alpha$ emission measure is expected due to a changing path length from the perspective of the observer as we move away from NGC 604. We note that given the distance of the slit to NGC 604, the gas disk would have to be thick enough to accommodate a sphere of 820 pc. Ultimately, this sort of geometry cannot be distinguished from other possibilities, such as the increase in the emission measure being due to a local feature like a filament. The ratio $[\mathrm{S} \mathrm{II}] / \mathrm{H} \alpha$ appears to increase with decreasing emission 
measure, and $[\mathrm{N} \mathrm{II}] / \mathrm{H} \alpha$ exhibits a slight increase with decreasing emission measure. The ratio of $[\mathrm{S} \mathrm{II}] /[\mathrm{N} \mathrm{II}]$ does not vary a great deal along the slit. This behavior of $[\mathrm{S} \mathrm{II}]$ and [N II] with emission measure and their overall elevated values with respect to $\mathrm{H}$ II regions is characteristic of DIG in other galaxies (e.g. Rand 1998 and Hoopes \& Walterbos 2003).

We were able to measure $[\mathrm{O} \mathrm{I}]$ in three of our seven apertures, and we calculated upper limits for the other four apertures. Our results indicate an increase of $[\mathrm{O} \mathrm{I}] / \mathrm{H} \alpha$ with decreasing emission measure. We measured $[\mathrm{O}$ III $] / \mathrm{H} \alpha$ in in every aperture, and the ratio stays fairly constant with emission measure. We were able to measure $[\mathrm{O}$ III $] / \mathrm{H} \beta$ in the first three apertures. The $\mathrm{H} \beta$ emission line fell near to the edge of the grating, where we had little sensitivity, and thus we were unable to detect $\mathrm{H} \beta$ in the regions of fainter emission. Our observed $\mathrm{H} \alpha / \mathrm{H} \beta$ ratio in the first three apertures is 3.1. Given the uncertainty in our measurements, this value is close enough to the intrinsic (unreddened) value of $\mathrm{H} \alpha / \mathrm{H} \beta=2.86$, so we did not do a correction for interstellar extinction. We used our observed $\mathrm{H} \alpha / \mathrm{H} \beta$ ratio to infer the $\mathrm{H} \beta$ intensities in the last four apertures. No correction was made for stellar absorption of the $\mathrm{H} \beta$ emission. Hoopes \& Walterbos (2003) found this correction in the DIG of M33 to be about 10\%, but this will vary depending on the contribution of the $\mathrm{H} \beta$ emission in the DIG relative to the $\mathrm{H} \beta$ absorption by the stars. Therefore, our measured [O III] $/ \mathrm{H} \beta$ ratios might be too high by $10 \%$, or possibly more. We had one tentative detection of He I in the first aperture with $\mathrm{He} \mathrm{I} / \mathrm{H} \alpha=0.033 \pm 0.019$. With the large uncertainty associated with this measurement, our detection does not improve upon the one in this region given by Hoopes \& Walterbos (2003), so we will not go into any further analysis of this result.

\section{2. [O I] Emission in the DIG}

Figure 3 shows our detection of [O I] $\lambda 6300$ in the first three apertures. The continuum levels adopted for the flux measurements are indicated on the spectra, and the noise was measured locally. This is the first detection of [O I] $\lambda 6300$ in the DIG of M 33, or any nonedge-on spiral other than the Milky Way. Our observed ratios of $[\mathrm{O} \mathrm{I}] / \mathrm{H} \alpha$ are 0.038, 0.040, and 0.097 for apertures 1,2 , and 3 respectively. These measurements were made in regions with emission measures of 48, 33, and $27 \mathrm{pc} \mathrm{cm}^{-6}$. For comparison, the WHAM facility measured $[\mathrm{O} \mathrm{I}] / \mathrm{H} \alpha$ in the warm ionized Galactic ISM in three directions (Reynolds et al. 1998). Two of their observations sampled DIG in the Galactic midplane, and the third at

$z=-300 \mathrm{pc}$. The observations sampled emission measures in the range of 6 to $24 \mathrm{pc} \mathrm{cm}^{-6}$ and they found $[\mathrm{O} \mathrm{I}] / \mathrm{H} \alpha=0.012-0.044$. The detection of $[\mathrm{O} \mathrm{I}]$ emission has implications regarding the relative contribution of shock ionization, so we will come back to this emission line in our comparison to photoionization models. 


\section{Comparison with Photoionization Models}

Hoopes \& Walterbos (2003) provide two CLOUDY photoionization models for M33: the "standard" DIG model and the "leaky H II region" model. The standard model simulates ionization by field OB stars by embedding OB stars in gas with the properties of DIG. The leaky H II region model is a combination of two models. The first model is of an H II region leaking a fraction of its ionizing photons. The ionizing spectrum of a star hardens as it passes through an H II region, thus the spectrum that emerges from the H II region is harder than the original stellar spectrum. As a result, H II region models with less leakage emit harder ionizing continua. The leaky H II region model then uses this hardened spectrum as the ionization source for a standard DIG model. In both models, they varied two parameters: the ionizing stellar temperature $T_{\star}$, and $q$, a measure of the ratio of the density of ionizing photons to the density of electrons. They varied the values of $q$ to bracket values suitable for DIG, which are thought to be about $\log q=-3$ to -4 . They used Kurucz (1991) ATLAS line-blanketed LTE stellar atmospheres for the ionizing continua and varied $T_{\star}$ from 30,000 to $50,000 \mathrm{~K}$. They used the abundances of He, O, N, and S from measurements made in NGC 604 (Vilchez et al. 1988). For the other elements, they used one-third the Orion Nebula abundances (the average of the factors suitable for oxygen and nitrogen).

Figure 4 compares the standard and leaky H II region model predictions with our observed line ratios. The $[\mathrm{O}$ III $] / \mathrm{H} \beta$ line ratios in apertures 4 through 7 have been inferred using our [O III] $/ \mathrm{Ha}$ measurements and our observed $\mathrm{H} \alpha / \mathrm{H} \beta$ ratio of 3.1. Two leaky $\mathrm{H}$ II region models are shown: one with $30 \%$ leakage from the region and one with $60 \%$ leakage. From this figure we find that, in general, the leaky H II region model with $30 \%$ leakage best fits our data. This conclusion is most clear in the middle and bottom plots in Figure 4, where the $30 \%$ leakage model with $\log q=-3$ to -4 brackets our observations and we have nearly constant inferred stellar temperatures of 40,000 to 42,000 K. In these two plots, our observed line ratios push the standard and $60 \%$ leakage models into the regime where $\log q=-2$, which is thought to be too large a value for $\log q$ in the DIG, and the inferred stellar temperatures are higher than those inferred by the $30 \%$ leakage model. In the top plot, at first glance we have several models that could be consistent with our observations. The hardened continuum that escapes from a leaky H II region produces higher $[\mathrm{N} \mathrm{II}] / \mathrm{H} \alpha$, $[\mathrm{S} \mathrm{II}] / \mathrm{H} \alpha$, and $[\mathrm{O} \mathrm{III}] / \mathrm{H} \beta$ ratios than the standard model. The $30 \%$ leakage models predict higher ratios for a given $q$ and $T_{\star}$ than the $60 \%$ leakage models. These differences are what ultimately make the $30 \%$ leakage model most consistent with our data in the top plot as well, where the increased $[\mathrm{N}$ II] $/ \mathrm{H} \alpha$ and $[\mathrm{S}$ II] $/ \mathrm{H} \alpha$ measurements in apertures 6 and 7 cannot be reproduced by the standard or $60 \%$ leakage models without extremely high stellar temperatures. The stellar temperature inferred by the $30 \%$ leakage model of about 42,000

$\mathrm{K}$ is reasonable for ionizing stars within an $\mathrm{H}$ II region. Our He I detection implies the 
presence of an O7 or earlier star, which would have a stellar temperature of about 40,000 $\mathrm{K}$ (Smith et al. 2002). We also note that 30\% leakage from H II regions is consistent with the study of the contribution of field OB stars to the ionization of the DIG given by Hoopes \& Walterbos (2000). Their study indicates that field stars only partially account for the ionization of the DIG, and that 25-30\% leakage from H II regions is necessary to account for the remaining DIG.

Hoopes \& Walterbos (2003) did not include [O I] in their diagnostic plots for leaky $\mathrm{H}$ II regions with multiple values for $\log q$, but they did include predictions for $[\mathrm{O} \mathrm{I}] / \mathrm{H} \alpha$ for $\log q=-3$ for various H II region leakage rates. For 30\% H II region leakage, our observed [O I] $/ \mathrm{H} \alpha$ ratios infer stellar temperatures between 38,000 and 42,000 K. For 60\% H II region leakage, our observed [O I] $/ \mathrm{H} \alpha$ ratios infer stellar temperatures between 30,000 and 44,000 K. Neither leaky H II region model requires unrealistic stellar temperatures to reproduce our observed $[\mathrm{O} \mathrm{I}] / \mathrm{H} \alpha$ ratios. Therefore, these observations, along with similar results for our [O III] observations, indicate that photoionization from leaky H II regions can comfortably provide the heating necessary to create the amount of $[\mathrm{O} \mathrm{I}]$ and [O III] emission that we detect. In this case, the candidate leaky H II region is NGC 604, a large H II region about $0.5 \mathrm{kpc}$ away from the slit. We have not considered shock ionization models here, and our emission line ratios may or may not be consistent with them. However, on energetic grounds, OB stars must be the dominant source for the ionization of the DIG, and our observations are consistent with pure photoionization models.

Based on observations obtained at the Gemini Observatory, which is operated by the Association of Universities for Research in Astronomy, Inc., under a cooperative agreement with the NSF on behalf of the Gemini partnership: the National Science Foundation (United States), the Particle Physics and Astronomy Research Council (United Kingdom), the National Research Council (Canada), CONICYT (Chile), the Australian Research Council (Australia), CNPq (Brazil) and CONICET (Argentina). 


\section{REFERENCES}

Abraham, R. G., et al. 2004, AJ, 127, 2455

Dopita, M. A. 1976, ApJ, 209, 395

Ferguson, A. M. N., Wyse, R. F. G., \& Gallagher, J. S. 1996, AJ, 112, 2567

Greenawalt, B. E. 1998, Ph.D. Thesis,

Greenawalt, B., Walterbos, R. A. M., Thilker, D., \& Hoopes, C. G. 1998, ApJ, 506, 135

Haffner, L. M., Reynolds, R. J., Tufte, S. L., Madsen, G. J., Jeans, K. P., \& Percival, J. W. 2003, ApJS, 149, 405

Hoopes, C. G., Walterbos, R. A. M., \& Greenwalt, B. E. 1996, AJ, 112, 1429

Hoopes, C. G., \& Walterbos, R. A. M. 2000, ApJ, 541, 597

Hoopes, C. G., \& Walterbos, R. A. M. 2003, ApJ, 586, 902

Kurucz, R. L. 1991, Precision Photometry: Astrophysics of the Galaxy, 27

Rand, R. J., Kulkarni, S. R., \& Hester, J. J. 1992, ApJ, 396, 97

Rand, R. J. 1998, ApJ, 501, 137

Reynolds, R. J. 1990, ApJ, 349, L17

Reynolds, R. J. 1991, IAU Symp. 144: The Interstellar Disk-Halo Connection in Galaxies, 144,67

Reynolds, R. J., Hausen, N. R., Tufte, S. L., \& Haffner, L. M. 1998, ApJ, 494, L99

Shull, J. M., \& McKee, C. F. 1979, ApJ, 227, 131

Slavin, J. D., Shull, J. M., \& Begelman, M. C. 1993, ApJ, 407, 83

Smith, L. J., Norris, R. P. F., \& Crowther, P. A. 2002, MNRAS, 337, 1309

Vilchez, J. M., Pagel, B. E. J., Diaz, A. I., Terlevich, E., \& Edmunds, M. G. 1988, MNRAS, 235, 633

Wang, J., Heckman, T. M., \& Lehnert, M. D. 1997, ApJ, 491, 114 


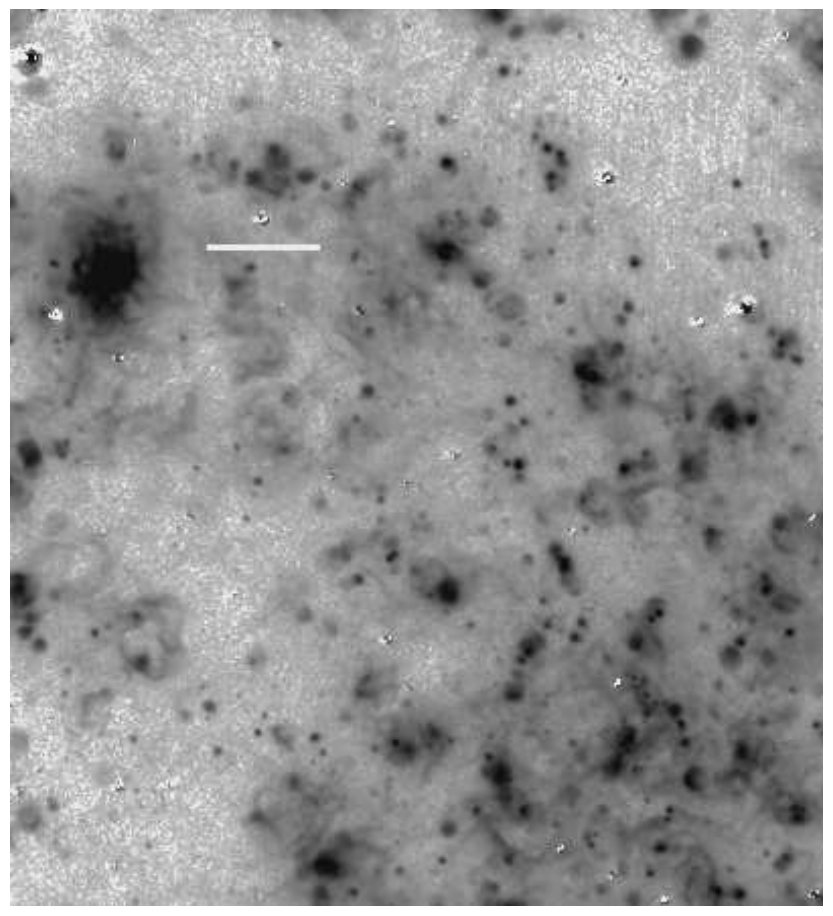

Fig. 1.- H $\alpha$ image of M33 showing the slit location. North is up and east is left. The slit is 108 " long, which at an assumed distance to M33 of $0.84 \mathrm{Mpc}$, corresponds to $440 \mathrm{pc}$. NGC 604 is the large, bright H II region to the east of the slit. The center of NGC 604 is 820 pc away from the west end of the slit. 


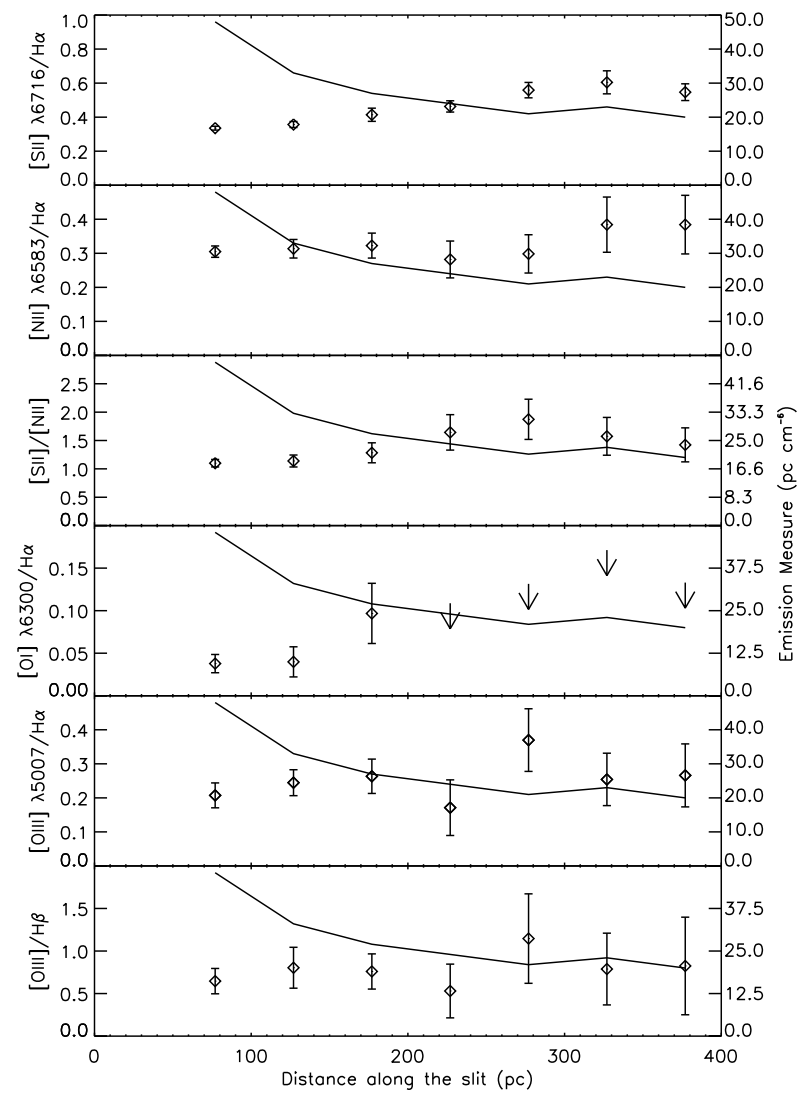

Fig. 2.- Observed emission line ratios along the slit. The first plot shows [S II] $\lambda 6716 / \mathrm{H} \alpha$, the second plot shows [N II] $\lambda 6583 / \mathrm{H} \alpha$, the third shows $[\mathrm{S} \mathrm{II}] /[\mathrm{N}$ II], the fourth shows [O I] $\lambda 6300 / \mathrm{H} \alpha$, the fifth shows [O III] $\lambda 5007 / \mathrm{H} \alpha$, and the last plot shows $[\mathrm{O}$ III] $/ \mathrm{H} \beta$. The $\mathrm{H} \beta$ fluxes in the last four apertures were inferred from our $\mathrm{H} \alpha$ flux measurements, using our average observed $\mathrm{H} \alpha / \mathrm{H} \beta$ ratio of 3.1 . 


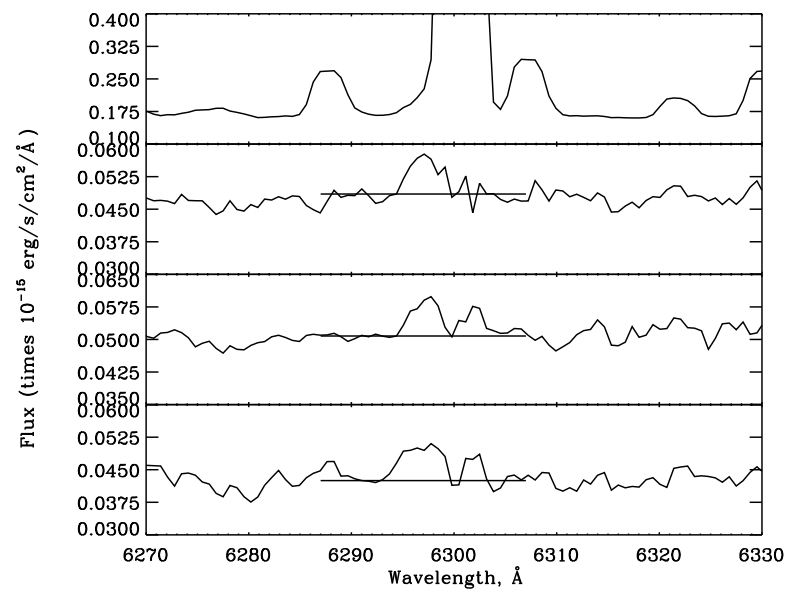

Fig. 3.- Spectrum of apertures 1, 2, and 3 in M33 around [O I]. The top plot shows the spectrum of aperture 1 before sky subtraction and the bottom three plots, apertures 1, 2, and 3 after sky subtraction. The continuum level used for each measurement is indicated. The feature at $6297 \AA$ is the [O I] emission from M33, and the feature to the right of it is the residual from the $[\mathrm{O} \mathrm{I}]$ airglow line subtraction. Note that the scale of the plot before sky subtraction is 10 times that of the plots after sky subtraction. 

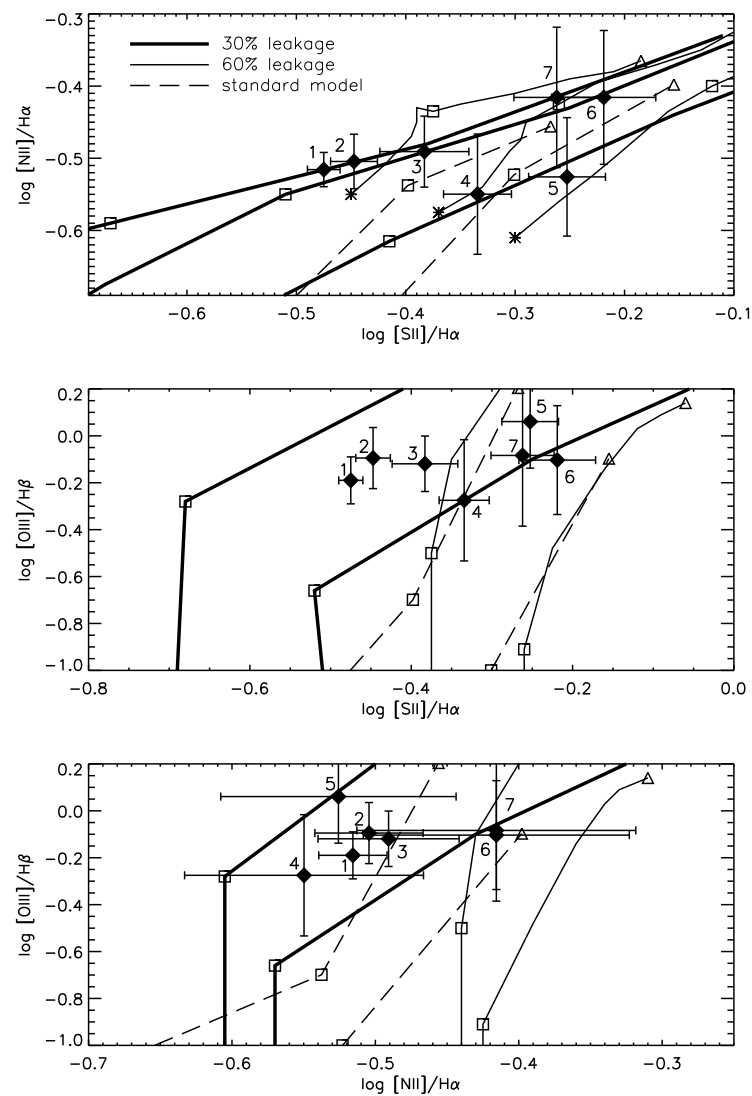

Fig. 4.- A comparison of photoionization models to our observed line ratios. The thick solid lines show the predictions for the $30 \% \mathrm{H}$ II region leakage models, the thin solid lines show the $60 \%$ leakage predictions, and the dashed lines shows the standard DIG model predictions. In the top plot we show three results for each model. From right to left, the lines indicate values of $\log q=-5,-4$, and -3 respectively. For the middle and bottom plot, from right to left, we show results for $\log q=-4$ and -3 . The asterisks indicate model stellar temperatures of 30,000 K; the squares, 40,000 K; and the triangles, 50,000 K. Our observed line ratios are plotted as solid diamonds. 